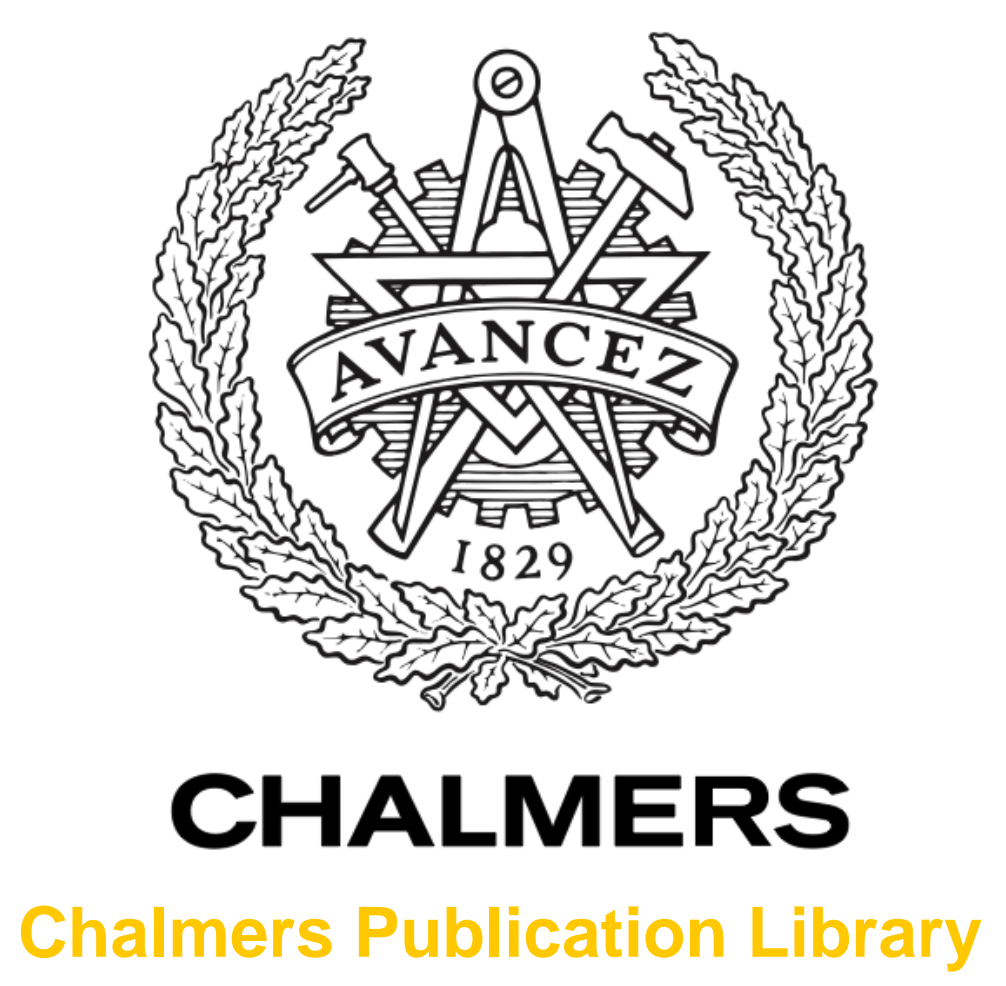

\title{
Requirements and test methods for vehicular antenna systems supporting cooperative \{TS applications1
}

This document has been downloaded from Chalmers Publication Library $(\mathrm{CPL})$. It is the author's version of a work that was accepted for publication in:

IEEE MTT-S International Conference on Microwaves for Intelligent Mobility (ICMIM)

Citation for the published paper:

Ström, E. ; Sjöberg, K. ; Carlsson, J. et al. (2015) "Requirements and test methods for vehicular antenna systems supporting cooperative $\{$ TS applications1". IEEE MTT-S

International Conference on Microwaves for Intelligent Mobility (ICMIM)

Downloaded from: http://publications.lib.chalmers.se/publication/220565

Notice: Changes introduced as a result of publishing processes such as copy-editing and formatting may not be reflected in this document. For a definitive version of this work, please refer to the published source. Please note that access to the published version might require a subscription. 


\title{
Requirements and test methods for vehicular antenna systems supporting cooperative ITS applications
}

\author{
Erik G. Ström*, Katrin Sjöberg ${ }^{\ddagger}$, Jan Carlsson ${ }^{\S}$, Amir Majidzadeh ${ }^{ף}$ \\ ${ }^{*}$ Chalmers Univ. of Technology, Dept. of Signals and Systems, erik.strom@chalmers.se; \\ $\ddagger$ Advanced Technology \& Research, Volvo Group Trucks Technology, katrin.sjoberg@volvo.com; \\ $\S$ SP Technical Research Institute of Sweden, Dept. of Electronics, jan.carlsson@sp.se; \\ ๆVolvo Car Corporation, amir.majidzadeh@ volvocars.com
}

\begin{abstract}
Antenna systems are crucial for the link performance of any wireless systems, including those supporting cooperative intelligent transport system (C-ITS) applications. It is therefore of great importance to define performance metrics that are relevant for C-ITS applications and a framework for measuring the metrics. In this paper, we propose to measure performance by cumulative distribution functions based on the output SNR of the antenna system under test. The SNR samples are collected with respect to the time scales relevant for C-ITS applications. The framework is suitable for both computer simulations and over-the-air measurements and can handle antenna systems that are time-varying and have multiple output ports.
\end{abstract}

\section{INTRODUCTION}

Cooperative intelligent transport systems (C-ITS) refers to systems in which vehicles exchange messages wirelessly to increase the awareness horizon of the driver beyond what can today be achieved with in-vehicle line-of-sight technologies such as radars and camera systems. Hence, C-ITS services are enabled by vehicle-to-vehicle (V2V) and vehicle-to-roadsideinfrastructure (V2I) wireless communication. This communication, collectively referred to as V2X communication, can either be direct between end nodes or indirect via intermediate nodes. The current state-of-the art for supporting direct communication, i.e., ITS-G5 in Europe and DSRC in the US [1], [2], are ad-hoc systems built on the IEEE 802.11p physical (PHY) and medium access control (MAC) layers. Cellular systems provide indirect communication, as (currently) all communication is done via a base station. It is foreseen that future vehicles will support both cellular and ad-hoc systems. Antenna systems are crucial for communication system performance and therefore also for C-ITS application performance. It is therefore of great importance to find appropriate antenna system performance metrics and methods for assessing these.

There are several possible performance assessment methods, all with their own merits and disadvantages. On one extreme, we have computer simulations in synthetic environments [3] and on the other extreme we have drive tests in real environments [4]. Somewhere in between, we have over-the-air (OTA) measurements in shielded or unshielded environments [5], [6]. It is likely that there is no one-size-fitsall solution. Computer simulations are very useful in the early stages of research and development, since many configurations

The research was partially funded by Swedish Governmental Agency for Innovation Systems (VINNOVA) within the VINN Excellence Center Chase project Antenna Systems for V2X Communication and FFI project Wireless Communication in Automotive Environment. and concepts can be tested with relative ease and low cost. OTA measurements are useful to further build confidence in smaller number of designs, while drive tests are necessary before fully committing to a design and commencing production.

In this paper, we will propose a framework that can be easily adapted to computer simulations and OTA measurements. The framework, which is similar in spirit to the methodology in [7], is less applicable to drive tests. Moreover, we will concentrate on systems for direct communication based on 802.11p. However, the concepts are, with minor modifications, more broadly applicable.

We will start by briefly describe C-ITS services and their requirements in Sec. II. In Sec. III, we explain how the propagation environment, antenna system, and receiver are modeled. The proposed antenna system performance metrics are defined in Sec. IV, and we summarize and draw conclusions in Sec. V.

\section{C-ITS APPLICATIONS}

During the last decade, it has been an intense activity within standardization on C-ITS both in the US and in Europe. A minimum set of protocols has been standardized on both continents to support the first generation of C-ITS applications. The artery of C-ITS is the position messages containing, e.g., speed, direction, and position of the vehicle, called basic safety messages (BSM) in the US and cooperative awareness messages (CAM) in Europe. The BSM and CAM development has been subject to certain harmonization efforts. The position messages will be broadcasted with an update rate of 1$10 \mathrm{~Hz}$, depending on the current data traffic load and vehicle dynamics.

In Europe, one additional message type has been developed called decentralized environmental notification message (DENM), which is triggered by C-ITS applications in case of a hazardous situation. Once triggered, DENMs can be periodically transmitted with an update rate of $1-20 \mathrm{~Hz}$ for a duration set by the application. The BSM also covers some event-triggered functionality, similar to that of DENM.

The US and European protocol stacks diverge in the network and transport layers. In Europe, the concept of GeoNetworking has been developed to support multihop communications and routing. DENMs can be subject to multihop communication whereas CAMs are always one-hop broadcast transmissions. In the US, a simple network and transport layer functionality is outlined in IEEE 1609.3, supporting one-hop broadcast communication. 
The European and US protocol stacks use the same PHY and MAC layers, as specified by IEEE 802.11p [8], which is a vehicular "profile" of the ubiquitous 802.11 WLAN standard. The amendment $802.11 \mathrm{p}$ is now classified as superseded and enrolled in the 2012 version of 802.11 [9]. Nevertheless, to facilitate reading of this paper, we will use the term $802.11 p$ when referring to the vehicular profile. The PHY is 802.11 orthogonal frequency division multiplexing (OFDM), as specified in [9, Clause 18] with the $10 \mathrm{MHz}$ channel spacing option.

Dedicated frequency bands have been set aside in North America $(5.850-5.925 \mathrm{GHz})$ and in Europe $(5.855-5.925 \mathrm{GHz})$ specifically for C-ITS with the aim to reduce the number of accidents and increase the road traffic efficiency [1], [2]. Radio propagation at the $5.9 \mathrm{GHz}$ band is challenging for wireless ad hoc communication at vehicular speeds. Large vehicles easily block the signal between sender and receiver and, therefore, the line-of-sight component is usually not available and the receiver has to rely on the multipath environment to enable reception of several replicas of the signal. Although no MIMO transmission schemes are part of 802.11 p, the receiver is free to use receive antenna diversity schemes to increase link stability.

The first generation of C-ITS applications (day one applications) intends to warn and guide the driver, and the driver is responsible for acting upon the received information. Applications aiming for increased road traffic safety include electronic emergency brake light (EEBL) mitigating shockwaves through traffic, traffic jam ahead, stationary vehicle warning, adverse weather conditions, etc. Green light optimal speed advisory (GLOSA) increases the traffic efficiency through signalized intersections by giving advice to the driver about appropriate speed for utilizing the "green wave."

Platooning and cooperative adaptive cruise control (CACC) are second generation C-ITS applications. Here, the vehicle is controlled automatically in both longitudinal and latitudinal directions based on in-vehicle sensor information together with received information from neighboring vehicles. Thanks to reduced air drag, platooning trucks can substantially reduce their fuel consumption and $\mathrm{CO}_{2}$ emissions. This is significant, as heavy duty vehicles are today the cause of up to $20 \%$ of all $\mathrm{CO}_{2}$ emissions on the planet [10]. Platooning is the C-ITS application that is putting up the possibly highest requirements on the communication in terms of delay and reliability. Excessive delays and jitter can violate the string stability of the platoon [11], and platooning vehicles will therefore exchange messages as often as 20 times per second. If reliability decreases below a predefined threshold, the whole platoon needs to be dissolved since safety cannot be guaranteed anymore. As with most other communication systems, uniformly distributed packet losses is not as serious as long bursts of packet losses. The latter will cause the disruption of C-ITS services as the length and probability of burst errors increases.

To summarize, C-ITS applications have the potential to increase safety and reduce environmental impact of the transport system. However, C-ITS services rely on timely delivery of quasi-periodic (BSM, CAM, DENM) messages. The latency, reliability, and data rate requirements vary between services. Some predictions state the future requirements as quite demanding: 1600 byte packets should be delivered within $5 \mathrm{~ms}$ with a probability of more than $99.999 \%$ [12]. However,

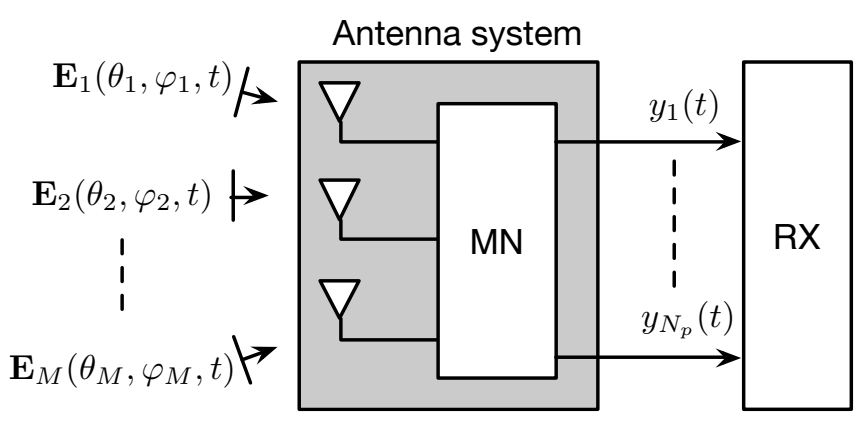

Fig. 1. Sketch of the considered system: $M$ plane waves incident on a number of antenna elements whose outputs are combined into $N_{p}$ receiver port signals.

today's CAM and BSM payloads are more modest, typical payloads will be around 400 byte including security overhead, and day one applications are more delay-tolerant. The payload size may vary depending on, e.g., the number of path history points of the vehicle that are included. The requirement on the delay has not yet been specified, but a rule of thumb is that the delay shall not exceed $100 \mathrm{~ms}$, which corresponds to the highest update rate of $10 \mathrm{~Hz}$

\section{SYSTEM MODEL}

We will consider antenna systems that consist of a number of antenna elements with a matching network (MN) and associated wiring, see Fig. 1. The antenna element outputs are processed by the $\mathrm{MN}$ into $N_{p}$ signals, where $N_{p}$ is the number of receiver $(\mathrm{RX})$ input ports. The port signals, $y_{n}(t)$ for $n=1,2, \ldots, N_{p}$ are determined by the MN properties, the antenna element embedded radiation patterns, and the incident electrical field. The receiver is not considered to be part of the device under test. In fact, we will assess the performance of the antenna system by measuring properties of the port signals.

\section{A. Time-scales}

The relevant time-scales for the system under study can be divided into symbol, frame, and packet repetition time scales ${ }^{1}$. The relation between these time scales and the other time variations, e.g., changes in propagation environment, vehicle dynamics, etc., are important when formulating meaningful performance metrics.

Timing parameters in 802.11 OFDM are decided by the channel spacing, which was selected to $10 \mathrm{MHz}$ early in the standardization process of DSRC and ITS-G5. Hence, the OFDM symbol time is $8 \mu \mathrm{s}$, including a $1.6 \mu \mathrm{s}$ long guard interval (cyclic prefix). CAM, BSM, and DENM messages are transmitted using QPSK modulation and rate $1 / 2$ convolutional coding, which yields a data rate of $6 \mathrm{Mbit} / \mathrm{s}$, excluding overhead for headers, pad bits, and convolutional code tail bits. In fact, it can be shown that an 802.11 frame has a minimum length of $48 \mu \mathrm{s}$, while a payload (MAC layer packet, i.e., the CAM, DENM, or BSM message plus protocol overhead)

${ }^{1}$ We will use "frame" and "packet" interchangeably to denote the portion of the transmitted signal that carries one CAM, BSM, or DENM message. 
of 400 byte have frame length $584 \mu$ s [9, Clause 18.3]. To summarize, for a C-ITS system using $802.11 \mathrm{p}$, the symbol time is $8 \mu \mathrm{s}$, the frame duration, $T_{f}$, is in the range $0.5-1 \mathrm{~ms}$ (depending on payload size), and the time between consecutive frames, $T_{p}$, is in the range $0.05-1 \mathrm{~s}$.

The channel variations can be divided into very slowly varying pathloss, slowly varying large-scale fading (LSF), and fast varying small-scale fading (SSF). Channel variations are dependent on mobility of transmitters, receivers, and scatterers and also of changes in the propagation environments (blocking vehicles, weather, and seasonal changes). Hence, SSF vary quite a lot over one frame duration in high-mobility scenarios, but can be assumed fixed over an OFDM symbol duration. The LSF is unlikely to change significantly between frames, except in special situations, e.g., when a vehicle suddenly appears in the line-of-sight. For simplicity, we will assume that pathloss and LSF effects are slow enough to be absorbed into the average received power, while the SSF effects are modeled (if desired) by the incident electrical field, as described in the following section.

\section{B. Propagation environment model}

We model the incident electrical field as a sum of plane waves, i.e., the complex field can be written as

$$
\mathbf{E}(\theta, \varphi, t)=\sum_{m=1}^{M} \mathbf{E}_{m}\left(\theta_{m}, \varphi_{m}, t\right)
$$

where $\theta_{m}$ and $\varphi_{m}$ is the elevation and azimuth angle of arrival of the $m$ th wave, respectively. In general, we allow for the complex field to be time-varying to take fading and Doppler shift into account.

The total power of the incoming waves is normalized and distributed in elevation and azimuth. Different mobility patterns and propagation environments, e.g., rural highway, urban street canyon, etc., will imply different polarizations, Doppler shifts, and power angular distributions. Hence, each important use case can be modeled by a certain propagation environment, parameterized by $\left\{\mathbf{E}_{1}\left(\theta_{1}, \varphi_{1}, t\right), \mathbf{E}_{2}\left(\theta_{2}, \varphi_{2}, t\right), \ldots, \mathbf{E}_{M}\left(\theta_{M}, \varphi_{M}, t\right)\right\}$, where some of the parameters are random. As an example, the propagation environment could be modeled with a single incoming plane wave with random, uniformly distributed polarization, random elevation and azimuth angles uniformly distributed in a solid angle sector $\left\{(\theta, \varphi): \theta \in\left[\theta^{\prime}, \theta^{\prime \prime}\right), \varphi \in\left[\varphi^{\prime}, \varphi^{\prime \prime}\right)\right\}$. This would be a reasonable model for a strong line-of-sight scenario with light traffic and negligible scattering. Another example would be to have a large number of incoming waves where the angle of arrival, power, polarization, and Doppler shift for each wave are derived from a geometry-based stochastic channel model, like the one in [13].

The propagation environment can fairly easily be implemented in an OTA setup, at least for a small $M$. For a computer tool, such as VIRM-lab [7], we can easily generate a large number of plane waves.

\section{Matching network model}

We will allow the matching network to have both passive and active components and to be time-varying. The time- variation is assumed to be slow compared to the frame duration, $T_{f}$, but not necessarily slow compared to the frame repetition time $T_{p}$. Allowing time-variations would cater for MNs that use, e.g., analog beamforming or beam-switching. In any event, we will model the $\mathrm{MN}$ as a linear, possibly timevarying, system.

The interface between the $\mathrm{MN}$ and the receiver consists of $N_{p}$ ports. Hence, the $\mathrm{MN}$ output is represented by $N_{p}$ complex, continuous-time signals, $y_{n}(t), n=1,2, \ldots, N_{p}$, which we model as $y_{n}(t)=x_{n}(t)+w_{n}(t)$, where $x_{n}(t)$ is the desired signal and $w_{n}(t)$ is complex additive noise, bandlimited to the communication signal bandwidth. We assume that the port signals are wide-sense stationary over at least a frame duration, but not necessarily over a frame repetition time. This to acknowledge that the expected signal power or noise power might change when the MN changes.

For the $n$th port signal, the noise power during the $k$ th frame is

$$
\sigma_{n}^{2}[k]=\mathbb{E}\left[\left|w_{n}(t)\right|^{2}\right], \quad t \in\left[k T_{p}, k T_{p}+T_{f}\right)
$$

and the signal power, time-averaged over the $k$ th frame, is

$$
p_{n}[k]=\frac{1}{T_{f}} \int_{k T_{p}}^{k T_{p}+T_{f}}\left|x_{n}(t)\right|^{2} d t .
$$

The port signal SNR during the $k$ th frame is therefore

$$
\gamma_{n}[k]=\frac{p_{n}[k]}{\sigma_{n}^{2}[k]} .
$$

Note that $p_{n}[k]$, and therefore also $\gamma_{n}[k]$, is a random variable due to the randomness in the propagation environment and the potential randomness in the MN.

\section{Receiver model}

The receiver is not considered to be a part of the antenna system (i.e., the system under test). Instead, we model the receiver as a black-box, whose performance is decided by the input SNRs, i.e., the port signal SNRs. In practice, the frame error rate, etc., depends on more signal properties, such as SNR variation over the time-frequency support of the transmitted frame. However, since it is cumbersome to describe all parameters that affect receiver performance, we will limit ourself to SNR here.

It should be noted that the receiver could be distributed over the vehicle, with analog frontends and A/D converters placed close the antenna elements. Nevertheless, the interface between the $\mathrm{MN}$ and $\mathrm{RX}$ is at the RX input ports.

As described above, the quality of the output signal from the antenna system is quantified by $\gamma_{n}[k]$, the SNR for receiver port $n$ averaged of the $k$ th frame, where frames are assumed to be spaced by $T_{p}$ seconds. Since we do not assume any particular processing by the receiver, we cannot fully specify the mapping between the port SNRs and the receiver performance, e.g., the probability of frame error. However, since 802.11p does not specify any spatial multiplexing schemes, we will assume that the receiver performs some type of diversity combining of the port signals. To proceed, we will consider two extreme diversity combining methods: selection 
combining (SC) and maximal ratio combining (MRC). The SNR after combining for the two methods are

$$
\begin{aligned}
\gamma_{\mathrm{SC}}[k] & =\max _{n} \gamma_{n}[k] \\
\gamma_{\mathrm{MRC}}[k] & =\sum_{n} \gamma_{n}[k] .
\end{aligned}
$$

From an SNR perspective, MRC is always better than SC, but it is also more costly to implement and the performance difference is not always significant. It is reasonable to assume that the combining scheme of any practical receiver would have an output SNR that is in between that of SC and MRC.

\section{Antenna Systems Performance Assessment}

In the following, we will develop performance metrics based on the SNR after combining. The metric definitions are not dependent on the combining method, and we let $\gamma[k]$ denote the post-combining SNR. The cumulative distribution function $(\mathrm{CDF})$ of $\gamma[k]$ is defined as

$$
P_{\gamma}(x) \triangleq \operatorname{Pr}\{\gamma[k] \leq x\} .
$$

Suppose we declare an outage when the SNR is less or equal to a certain outage SNR $\gamma_{o}$. The outage probability is then simply $P_{\gamma}\left(\gamma_{o}\right)$. The outage SNR will be depend on the receiver quality and combining strategy as well as on the time-dispersion and time-variations of the wireless channel. Hence, the outage SNR depends partly on the propagation environment. For these reasons, we argue that the full CDF should be considered as the performance metric.

The number of consecutive outage events is also of interest, as this is related to the time between two correctly received packets, i.e., the received inter-packet spacing $\tau_{\text {IP }}$. To see this, let us assume that the $k$ th frame can be correctly decoded if and only if $\gamma[k] \geq \gamma_{o}$. This is an approximation, as the frame error event is not exactly the same as the outage event-frames can be lost also at high SNRs and frames can be decoded also at low SNRs.

Let $\mathcal{K} \triangleq\left(k: \gamma[k] \geq \gamma_{o}\right)$ be a list of all successfully decoded frames, sorted in ascending order. Suppose the list has $L$ entries and that the $l$ th entry is denoted by $\mathcal{K}[l]$. Then the time between two successfully decoded frames is

$$
\tau_{\mathrm{IP}}=T_{p}(\mathcal{K}[l]-\mathcal{K}[l-1]), \quad l=2,3, \ldots, L .
$$

Clearly, $\tau_{\mathrm{IP}}$ is a discrete random variable, which is fully characterized by its $\mathrm{CDF}$

$$
P_{\tau_{\text {IP }}}(x) \triangleq \operatorname{Pr}\left\{\tau_{\text {IP }} \leq x\right\}
$$

The received inter-packet spacing CDF can be used in a similar way as the SNR CDF. Given that the inter-packet spacing should be less or equal to $\tau_{o}=l_{\max } T_{p}$ for the $\mathrm{C}$ ITS application to function as intended, the application outage probability is $\operatorname{Pr}\left\{\tau_{\mathrm{IP}}>l_{\max } T_{p}\right\}=1-P_{\tau_{\mathrm{IP}}}\left(l_{\max } T_{p}\right)$.

\section{SUMMARY AND CONCLUSION}

To measure or simulate the performance of an antenna system, we believe that the propagation environment can be adequately modeled by a number of incident plane waves. The parameters of the waves, i.e., relative power, polarization, angle of arrival, Doppler shift, etc., are either fixed or random. This modeling is relatively convenient for both computer simulations and over-the-air (OTA) measurements. The definitions of the environments are left for future work, but should be based on relevant use cases for C-ITS applications and channel measurements.

We have argued that performance of an antenna system can be quantified in two CDFs, $P_{\gamma}(x)$ and $P_{\tau_{\text {IP }}}(x)$, defined in (4) and (5), respectively. Multiport antenna systems can be handled by combining the port SNRs with selection combining and maximal ratio combining, see (2) and (3), as the post-combining SNR of most practical combining schemes will fall in between SC and MRC. Hence, for multiport antenna systems, we will measure performance by four CDFs, $\left\{P_{\gamma}(x), P_{\tau_{\mathrm{IP}}}(x)\right\}$ for $\gamma[k]=\gamma_{\mathrm{SC}}[k]$ and $\gamma[k]=\gamma_{\mathrm{MRC}}[k]$.

Furthermore, we have argued that the statistics should be collected with respect to the time-scales that are relevant for CITS systems. That is, we collect SNR samples averaged over one frame duration (approx. $1 \mathrm{~ms}$ ) and spaced by the frame repetition time (approx. $100 \mathrm{~ms}$ ).

\section{REFERENCES}

[1] J. B. Kenney, "Dedicated short-range communications (DSRC) standards in the United States," Proc. IEEE, vol. 99, no. 7, pp. 1162-1182, Jul. 2011, invited paper.

[2] E. G. Ström, "On medium access and physical layer standards for cooperative intelligent transport systems in Europe," Proc. IEEE, vol. 99, no. 7, pp. 1183-1188, Jul. 2011, invited paper.

[3] L. Reichardt, J. Pontes, W. Wiesbeck, and T. Zwick, "Virtual drives in vehicular communication," IEEE Veh. Technol. Mag., vol. 6, no. 2, pp. 54-62, May 2011.

[4] L. Y. Ekiz, "Vehicular service delivery via hybrid access and antennas," Ph.D. dissertation, Vienna Univ. of Technology, 2014. [Online]. Available: http://permalink.obvsg.at/AC12086481

[5] R. Sharma, C. Schneider, W. Kotterman, G. Sommerkorn, P. Große, F. Wollenschläger, G. Del Galdo, M. Hein, and R. Thomä, "Over-theair testing of car-to-car and car-to-infrastructure communication in a virtual electromagnetic environment," in Proc. Annual Conf. IEEE Ind. Electron. Soc., Nov. 2013, pp. 6897-6902.

[6] M. Nilsson, P. Hallbjörner, N. Araback, B. Bergqvist, and F. Tufvesson, "Multipath propagation simulator for V2X communication tests on cars," in Proc. Eur. Conf. Ant. and Prop. (EuCAP), Apr. 2013, pp. 1342-1346.

[7] E. Condo Neira, U. Carlberg, J. Carlsson, K. Karlsson, and E. G. Ström, "Evaluation of V2X antenna performance using a multipath simulation tool," in Proc. Eur. Conf. Ant. and Prop. (EuCAP), Apr. 2014.

[8] Wireless LAN Medium Access Control (MAC) and Physical Layer (PHY) Specification: Amendment 6: Wireless Access in Vehicular Environments, IEEE Std. 802.11p, 2010.

[9] Wireless LAN Medium Access Control (MAC) and Physical Layer (PHY) Specification, IEEE Std. 802.11, 2012.

[10] S. Kahn Ribeiro, S. Kobayashi, M. Beuthe, J. Gasca, D. Greene, D. S. Lee, Y. Muromachi, P. J. Newton, S. Plotkin, D. Sperling, R. Wit, and P. J. Zhou, Climate Change 2007: Mitigation. Cambridge University Press, 2007, ch. 5, Transport and its Infrastructure. [Online]. Available: http://www.ipcc.ch/pdf/assessment-report/ar4/wg3/ ar4-wg3-chapter5.pdf

[11] X. Liu, A. Goldsmith, S. S. Mahal, and J. K. Hedrick, "Effects of communication delay on string stability in vehicle platoons," in IEEE Intell. Transp. Systems Conf., Aug. 2001, pp. 625-630.

[12] "Scenarios, requirements and KPIs for 5G mobile and wireless system," Apr. 2013, ICT-317669-METIS/D1.1, METIS Deliverable D1.1. [Online]. Available: https://www.metis2020.com/documents/deliverables/

[13] J. Karedal, F. Tufvesson, N. Czink, A. Paier, C. Dumard, T. Zemen, C. Mecklenbräuker, and A. Molisch, "A geometry-based stochastic MIMO model for vehicle-to-vehicle communications," IEEE Trans. Wireless Commun., vol. 8, no. 7, pp. 3646-3657, Jul. 2009. 